\title{
THE PRESENT STATUS OF THE THEORY OF ELECTROLYTES*
}

\section{CHARLES A. KRAUS}

1. Introduction. It is not generally recognized that physical research is quite as dependent upon aids to the mind as it is upon aids to the senses. Indeed, it is the aids to the mind that are fashioned with greatest difficulty; only at long intervals does there appear a figure possessing that high degree of originality necessary for the construction of a new mental device that may be used successfully in analyzing the complex phenomena of nature. In chemistry, we have three such devices, namely: thermodynamics, statistical mechanics, and quantum mechanics. For the first two of these we are largely indebted to Josiah Willard Gibbs. Although a mathematician by training, Gibbs possessed that intuitive physical sense which enabled him to reduce physical phenomena to their lowest terms. His contributions to chemical science rank in importance with those of Lavoisier and Dalton.

To the layman it must appear that the rapid progress of modern science is due to experimentation. Nothing could be farther from the truth. In all ages, there have been men who experimented, just as there have been those who philosophized; but their contributions to scientific knowledge were few in number and limited in scope. What distinguishes our present age from all others is that experiment and theory have been joined in complementary fashion. The achievements of science have been due to this happy combination.

The subject that I propose to discuss is a small segment of a much broader one, namely, the relation between electricity and matter. While the problem of electrolytes is but a fraction of the broader problem, its study has led to the development of concepts which have made it possible to interpret matter in all its forms in terms of electrically charged particles. $\dagger$

Interesting and instructive as it might be, an historical introduction is not possible on this occasion. Suffice it to say that the problem

\footnotetext{
* The fourteenth Josiah Willard Gibbs Lecture, delivered at Indianapolis, Indiana, December 28, 1937, under the auspices of the American Mathematical Society, at a joint meeting of the Society with the American Association for the Advancement of Science.

$\dagger$ This excepts the neutron, the relation of which to other types of ultimate particles of matter is as yet little understood.
} 
of electrolytes had its inception in the observations of chemists, several hundred years ago, and that on the physical side it received its first impetus through the discoveries of Volta. These were followed by the epoch-making investigations of Faraday, who laid the foundations of the theory of electrolytes. He showed that matter and electricity are related in a definite, quantitative manner, whatever the nature of the atoms concerned. Most important, he introduced the concept of an ion as a charged atom or molecule. He accounted for the passage of electricity through a solution of an electrolyte by the motion of oppositely charged ions through the solution.

The ionic concept is one of the most important that has ever been introduced into physical science. It is more fundamental than the concept of atoms and molecules, for we now know that all atoms are composed of positively charged nuclei, surrounded by negative electrons equal in number to the charge on the nucleus. In final analysis, all matter is constructed of assemblies of ions. Today, the structure of the nucleus itself is interpreted in terms of ionic phenomena.

The application of the ionic concept to the problem of the structure of matter is a by-product of the problem of electrolytes. This latter problem is still far from solution in complete form. Through the work of Clausius, Hittorf, Kohlrausch, Arrhenius, and others, it finally became clear that, although electrolytes contain oppositely charged ions, these electrolytes may dissociate into their constituent ions which move about freely in the solvent medium. Arrhenius assumed an equilibrium between the free ions and the undissociated molecules of an electrolyte and sought to account for the observed properties of such a solution through displacement of this equilibrium. While his theory was successful in accounting for solutions of weak electrolytes, it led to contradictions in the case of strong electrolytes. These contradictions were not resolved until Debye introduced his theory of ion interaction some fifteen years ago.

2. Ion interaction: thermodynamic properties. The properties of electrolytic solutions fall into two classes: (1) those of reversible and (2) those of irreversible processes. The first class includes all thermodynamic properties such as freezing point, boiling point, vapor pressure, and the various thermodynamic energy functions; the second includes all time-dependent phenomena, such as conductance, diffusion, and transference.

According to Gibbs, equilibrium in a multiphase system requires that the thermodynamic potential of any component shall have the same value in the several phases. In systems in which reaction occurs and equilibrium exists among the reactants, the sum of the thermo- 
dynamic potentials of the reactants must be equal to zero. It becomes necessary, therefore, to determine how the thermodynamic potential of an electrolyte depends upon the various thermodynamic variables.

In a dilute solution of a substance between whose molecules no forces act (an ideal solute), the thermodynamic potential of the solute varies as the logarithm of the concentration; thus

$$
\mu_{i}=R T \log C+I,
$$

where $\mu_{i}$ is the thermodynamic potential of the ideal solute, $R$ the gas constant, $T$ the absolute temperature, $C$ the concentration in moles per liter, and $I$ an integration constant. If forces act between the molecules of the solute, then the observed thermodynamic potential becomes

$$
\mu_{0}=R T \log C+R T \log f+I,
$$

where $R T \log f$ is the energy due to the interacting forces between the molecules. It is evident that this energy is $R T \log f=\mu_{0}-\mu_{i}$.

If we have an electrolyte $A B$ which is in equilibrium with its ions $A^{+}$and $B^{-}$according to the reaction equation $A^{+}+B^{-} \rightleftarrows A B$, then, according to the theory of Arrhenius, we should have the equilibrium equation

$$
\frac{C_{A^{+}} \times C_{B}^{-}}{C_{A B}}=K,
$$

where $K$ is the equilibrium constant. This equation is based on the assumption that no forces act between the ions, between the ions and the undissociated molecules $A B$, or between the molecules $A B$ themselves. In the light of our preceding discussion, this assumption is not permissible, since the Coulomb forces, which vary as the inverse square of the distance, are constantly acting between the ions. The difficulty may be resolved by taking into account the energy due to the interaction between the ions. For a binary electrolyte, this energy term is written in the form $R T \log f^{2}$, in which case our equilibrium equation assumes the simple form

$$
\frac{C_{A}^{+} \times C_{B}^{-}}{C_{A B}} f^{2}=K .
$$

If $\gamma$ is the fraction of the electrolyte dissociated into ions, then $C_{A}{ }^{+}=C_{B}^{-}=C \gamma$, and $C_{A B}=C(1-\gamma)$. Hence, our equation takes the form

$$
\frac{C \gamma^{2}}{1-\gamma} f^{2}=K
$$


If we know the value of $f$ (the activity coefficient), it becomes possible to calculate the equilibrium constant $K$, provided $\gamma$ is known.

Let us first consider the case of a completely dissociated electrolyte, $(\gamma=1)$, at relatively low concen tration; the positive and negative ions will be distributed throughout the solvent medium in a somewhat random manner due to their energy of thermal agitation. This distribution of the ions, however, will not be the same as that of uncharged molecules between which no forces act. Due to the Coulomb forces acting between the ions, there is a greater probability that a positive charge will be found in the neighborhood of a negative charge than in the neighborhood of another positive charge, and the same is true of a negative charge in the neighborhood of a positive charge.

Debye has succeeded in computing the charge distribution in the neighborhood of an ion. A detailed account of the method employed would be out of place here, but I may briefly present the physical picture and give the final result. Let us fix our thoughts for the moment on a positive ion bearing a unit charge; it will, on the average (with respect to time) be surrounded by one unit of negative charge, distributed symmetrically with respect to the central ion, and the amount of charge in a shell of thickness $d r$, around the positive charge, will vary in a regular manner with the distance from the central charge. Actually, the charge in a shell of given thickness gradually increases from very low values, passes through a maximum, and then approaches zero at great distances. According to the Debye theory, the charge density falls off to $1 / \epsilon$ th its value at a distance $1 / \kappa=1.99 \times 10^{-10}(D T / C)^{1 / 2}$, where $D$ is the dielectric constant of the medium. As the concentration diminishes, the distance $1 / \kappa$ increases, and it follows that, in diluting a solution, work must be done against the forces acting between the central ion and its surrounding charge, or its ion atmosphere, as it is called.

For a relatively dilute solution, the equation for the activity coefficient of the ions takes the simple form

$$
-\log _{10} f=\beta C^{1 / 2},
$$

where

$$
\beta=\frac{1.81 \times 10^{6}}{(D T)^{3 / 2}} .
$$

In our equations above, we have assumed that each ion carries a unit charge. Similar relations are easily obtained for the case of ions with any number of charges. For the sake of simplicity, our subsequent discussion will be restricted to uni-univalent salts, unless otherwise mentioned. 
In Fig. 1 are plotted values of $-\log f$ against the square root of the (total ion) concentration for uni-univalent, uni-divalent, and uni-trivalent salts. As may be seen, at lower concentrations the points ap-

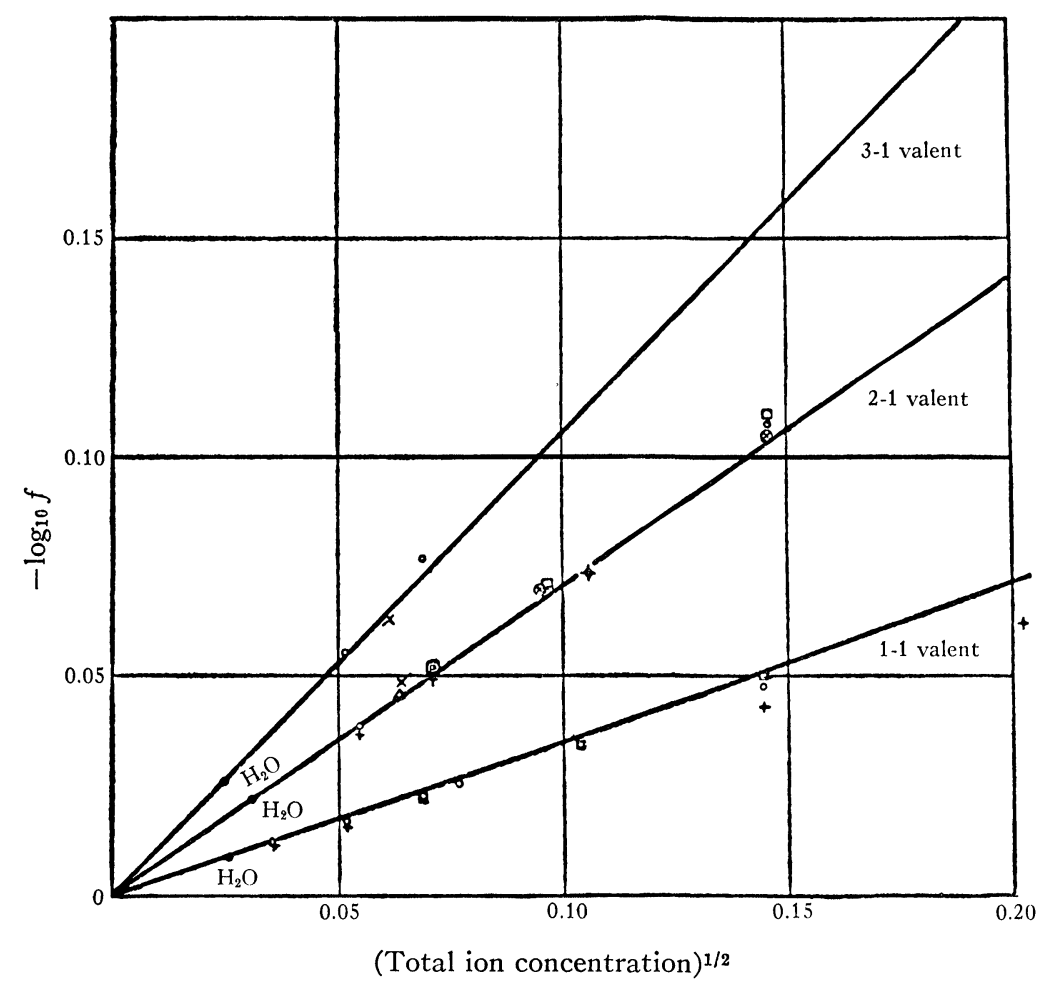

FIG. 1

Plot of $-\log f$ as a function of the square root of total ion concentration for salts in water.

proximate closely to the straight lines required by theory. As the charge on one of the ions increases, the curves become steeper in accord with theory. The equation has been tested with the very extensive experimental material relating to aqueous solutions and has been found to hold over a considerable range of concentration in the dilute region. At high concentrations, however, the deviations become large.

3. The Debye-Hückel-Onsager equation. Let us now turn to the irreversible phenomenon of the electrical conductance of a solution of an electrolyte. This is an important case, since the conductance is the one property of electrolytic solutions that may be measured with high precision over the widest range of conditions. It is known that 
in the case of all strong electrolytes the equivalent conductance $\Lambda$ (the specific conductance divided by the equivalent concentration) increases with decreasing concentration and approaches a limiting value. Arrhenius accounted for the gradually decreasing conductance at higher concentration by assuming an equilibrium between ions and undissociated molecules, the equilibrium being shifted with increasing concentration in the direction of a larger proportion of undissociated molecules. Debye and Hückel, however, showed that the decreased conductance at higher concentration is due to a decrease in the mobility of the ions themselves. The reason for this is not difficult to understand.

The force acting on an ion depends not only upon the external field under which it tends to move, but also upon the field due to the ion atmosphere. In the case of a stationary ion, the ion atmosphere is symmetrical with respect to the central charge, and the resultant force acting on the charge is zero. With a moving ion, however, the ion atmosphere becomes unsymmetrical with respect to the central charge and exerts upon this charge a force that counteracts the applied field and thus tends to reduce the mobility of the ion. The asymmetry of the ion atmosphere of a moving charge is due to the fact that it requires a finite time to build up the ion atmosphere about a charge and, similarly, it requires a finite time for the ion atmosphere about a charge to become dissipated when the central charge disappears. This time is called the relaxation time and may be computed from known constants of the electrolyte and of the medium in which it is dissolved. Since the diameter of the ion atmosphere $(1 / \kappa) \mathrm{di}$ minishes with increasing concentration, the asymmetry of the charge (in the ion atmosphere) exerts an increasingly great influence upon the moving ion as the concentration increases.

Another factor needs to be taken into account, namely, what is known as the electrophoretic effect. Due to the charge of the ion atmosphere, the water molecules in this atmosphere are carried in a direction opposite to that in which the ion moves. This results in a further diminution in the forward movement of the central charge.

The influence of the ion atmosphere on the conductance of an electrolyte, as computed by Debye and Hückel and Onsager, takes the comparatively simple form of the following equation:

$$
\Lambda=\gamma\left(\Lambda_{0}-\alpha\left({ }_{2} C\right)^{1 / 2}\right),
$$

where $\alpha=8.18 \times 10^{5} /(D T)^{3 / 2} \Lambda_{0}+82 / \eta(D T)^{1 / 2}$. Here $\eta$ is the viscosity of the solution, and $\Lambda_{0}$ is the limiting value which the equivalent conductance approaches as the concentration is diminished without 
limit; in other words, it is the conductance of the ion when the influence of the ion atmosphere becomes vanishingly small.

In a solution of a strong electrolyte in a solvent of high dielectric constant (such as water), the electrolyte is completely dissociated, and $\gamma$, in the above equation, becomes equal to unity. In the equations originally developed by Debye and Hückel, $\gamma$ was assumed to be unity, and the correctness of this assumption has been amply confirmed by the experimental facts in the case of strong electrolytes. In the case of weak electrolytes, however, it is necessary to introduce the degree of dissociation $\gamma$.

How well the Debye-Hückel-Onsager theory accounts for the conductance of solutions of electrolytes in water is illustrated in Fig. 2,

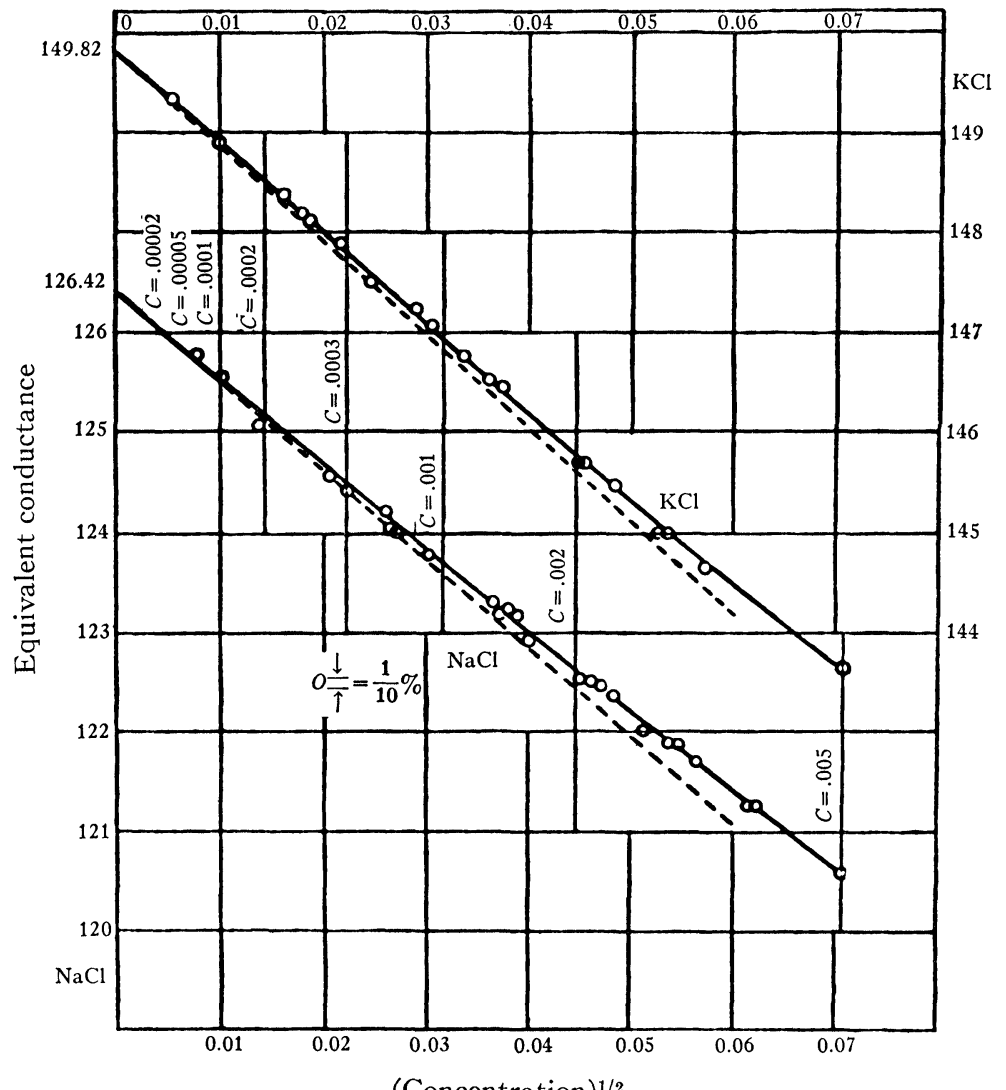

(Concentration) $)^{1 / 2}$

FIG. 2

Plot of equivalent conductance as a function of square root of concentration for $\mathrm{NaCl}$ and $\mathrm{KCl}$ in water at $25^{\circ} \mathrm{C}$. 
where the equivalent conductance of aqueous solutions of potassium chloride and sodium chloride are plotted against the square root of concentration. As may be seen from the figure, the experimental points lie upon curves that approach definite limiting tangents, shown in the figure as broken lines. These tangents, as drawn, are determined by the coefficients $\alpha$ and are, therefore, not arbitrary. It is evident that, while the Debye-Hückel-Onsager equation gives a correct interpretation of the facts in very dilute solutions, at higher concentrations, even as low as $0.001 \mathrm{~N}$, the deviations become appreciable and become increasingly larger as the concentration increases.

4. Ion association. As stated above, for solutions of weak acids and bases it is necessary to assume incomplete dissociation, even in aqueous solution. In other words, for these electrolytes the ions interact at short distances to form neutral molecules. There is reason for believing that in the case of acids and bases something more is involved than merely the Coulomb forces acting between the ions. For normal electrolytes (which are strong electrolytes in water) dissolved in solvents of lower dielectric constant, we have good reason to believe that association of ions occurs due to Coulombic interaction at short distances. Bjerrum considered this problem from the theoretical point of view as early as 1926 . He showed that in solvents of lower dielectric constant the distribution of ions of one charge in the neighborhood of an ion of opposite charge takes the form shown in Fig. 3. (The figure is due to Dr. Fuoss.) Here there is a high concentration of ions within one or two ion-diameters of the central ion, then a minimum in the distribution curve, and thereafter a maximum similar to that in the ion atmosphere of strong electrolytes in water. If it is assumed that an ion lying near to the central ion takes no part in the conduction process, while an ion at a distance exerts its normal influence in the ion atmosphere, and that there is an equilibrium between the two types according to the law of mass action, then the dissociation constant of the electrolyte may be related to the dielectric constant of the solvent medium and the sum of the radii of the two ions. The complete expression is somewhat complex, but a first approximation takes the form

$$
\frac{1}{K}=\frac{4 \pi N}{1000} a^{3} \frac{a D k T}{e^{2}} \exp \left(\frac{e^{2}}{a D k T}\right),
$$

where $N$ is Avogadro's number, $k$ is Boltzmann's constant, $e$ is the unit charge, and $a$ is the distance between the centers of charge of the 
two ions when in contact. It is seen from this equation that the dissociation constant $K$ is closely related to the dielectric constant $D$ of the medium, on the one hand, and to $a$, the distance of closest ap-

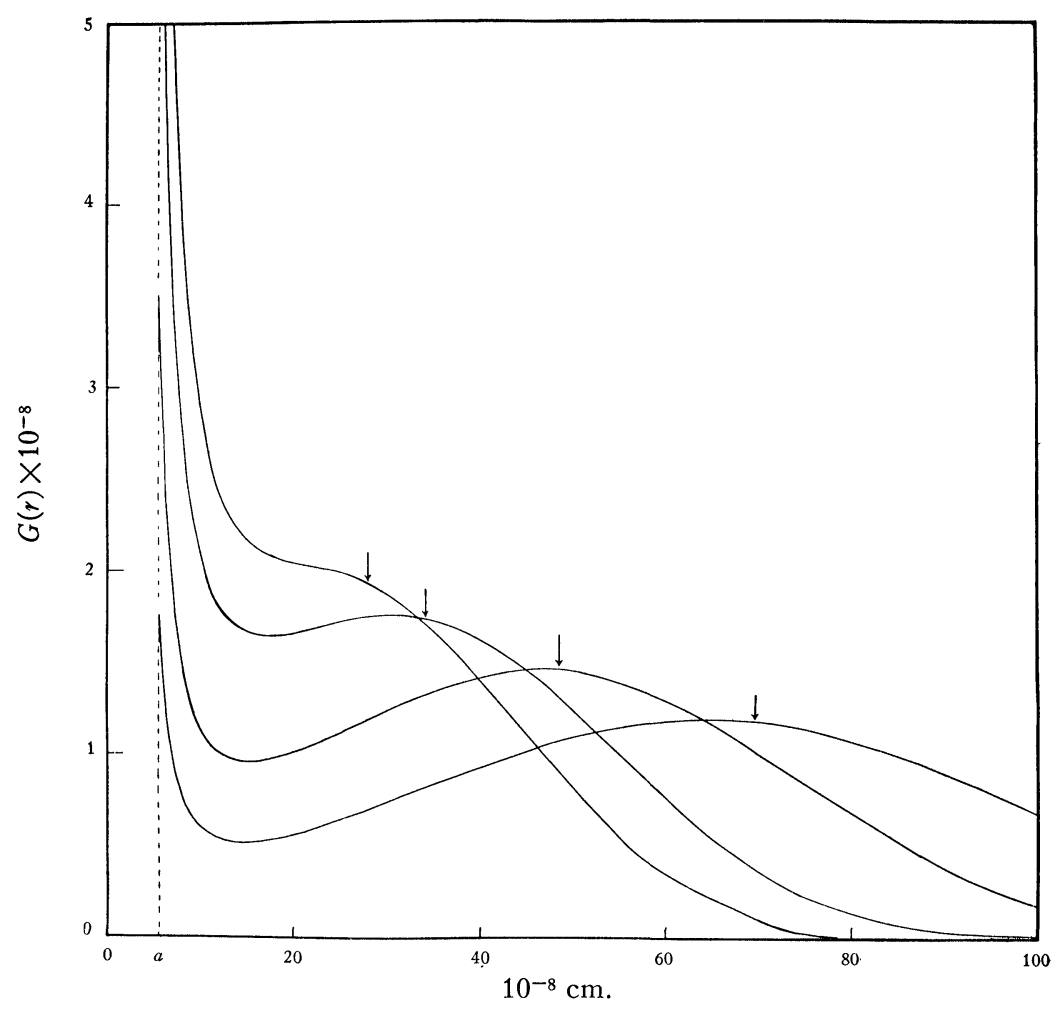

FIG. 3

Distribution of charge around central ion in solvent of dielectric constant $D=20 . a=5.57 \times 10^{-8} . C=0.5,1,2,3 \times 10^{-3}$.

proach of the ions, on the other. It is evident that the value of $K$ diminishes rapidly with decreasing value of the distance $a$, or with decreasing value of the dielectric constant $D$.

In Fig. 4 is shown the curve connecting $-\log K$ with the dielectric constant. The curve is based on the value $a=6.4 \times 10^{-8} \mathrm{~cm}$. and the circles represent experimental points for solutions of tetraisoamylammonium nitrate in mixtures of dioxane and water. As may be seen from the figure, the dissociation constant reaches values approaching unity for a value of $D$ in the neighborhood of forty. For this and higher values of $D$, the electrolyte is completely dissociated. For $D=2.4$ the dissociation constant has fallen to $2 \times 10^{-16}$. 
We should expect that all electrolytes in solvents of lower dielectric constant would exhibit marked association of their ions, and that the association would be the greater the smaller the ions. This has been amply confirmed by the work of Dr. Fuoss and others in the Brown laboratories.

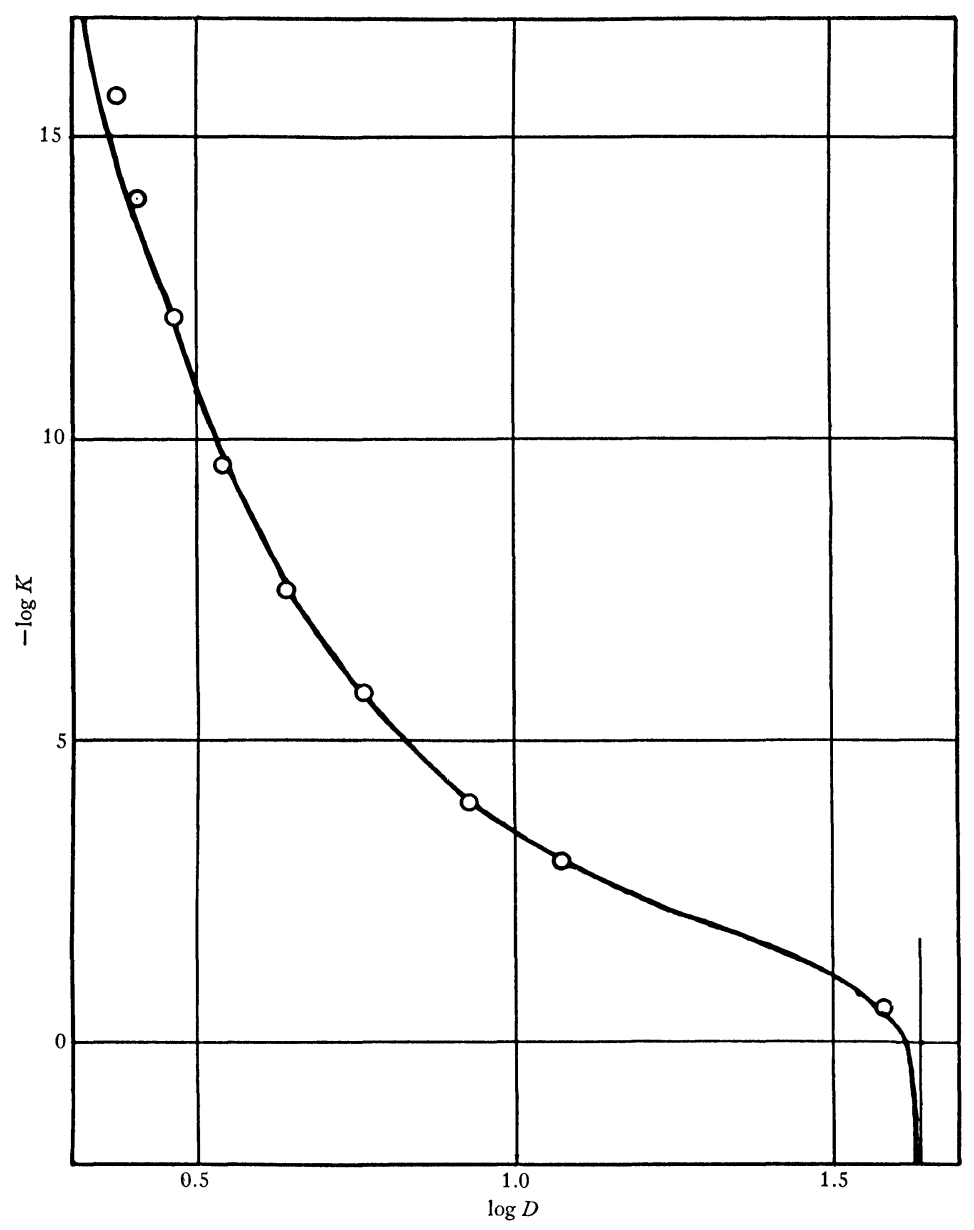

FIG. 4

Plot of $-\log K$ as function of $\log D . \quad a=6.4 \times 10^{-8}$.

In examining experimental conductance data in the light of the above theoretical considerations, it is necessary to solve simultaneously equations (1), (2), and (3) above. While an explicit solution is not possible, Dr. Fuoss has developed ready methods of approxima- 
tion that render it possible to evaluate the experimental results in terms of the theory. Essentially, what is necessary, according to the present theory, is to take into account the equilibrium between free and associated ions according to the law of mass action, and the long range interaction of the ions between one another in terms of the ionatmosphere effect. How well the theory accounts for the experimental values is shown in Fig. 5, where are plotted values of $\Lambda_{0} F / \Lambda$ against those of $C \Lambda f^{2} / F$, where $f$ is the activity coefficient of the ions and $F$ is a function readily computed from conductance data (Fuoss, Journal of the American Chemical Society, vol. 57 (1935), p. 488).

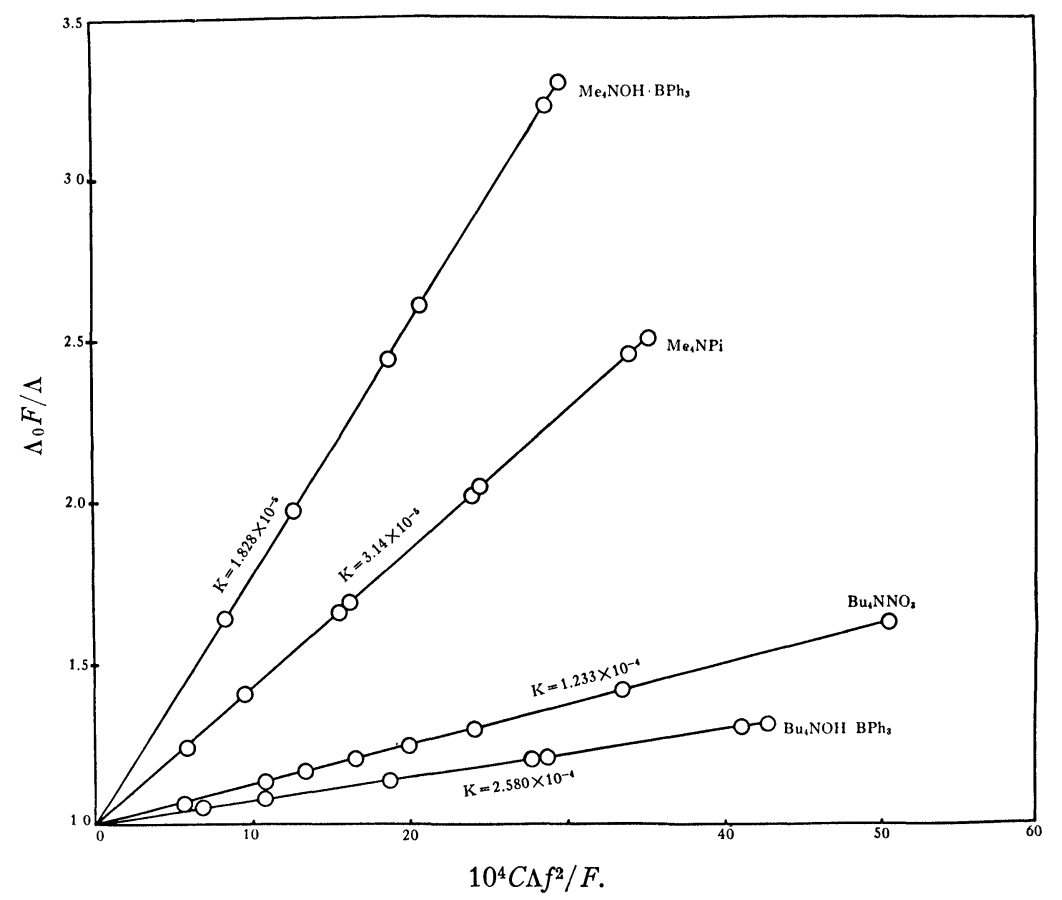

FIG. 5

Plot of conductance function for salts in ethylene chloride.

As may be seen from the figure, the experimental points closely approximate the linear relation required according to theory. At higher concentrations, however, the deviation becomes increasingly larger. Again, theory supplies a satisfactory limiting solution.

For solutions of electrolytes having spherical ions, the dissociation constant is determined by $a$, the sum of the ion radii, and $D$, the dielectric constant of the solvent medium. If $K$ is known, $a$ may be 
computed therefrom. The values of $a$ so obtained are in reasonable accord with atomic dimensions as determined by other means. With electrolytes having complex or unsymmetrical ions, the distance $a$ depends upon the relative position of the ions and the simple theory (based on spherical ions) becomes an approximation. Nevertheless, the experimental values are fairly well accounted for even in this case.

In Table 1 are given values of $\Lambda_{0}, K$, and $a$ for a number of electrolytes in liquid ammonia.

TABle 1

Conductance of salts in liquid ammonia

\begin{tabular}{lccc}
\multicolumn{1}{c}{ Salt } & $\Lambda_{0}$ & $K \times 10^{4}$ & $a \times 10^{8}$ \\
$\mathrm{LiNO}_{3}$ & 277 & 36.5 & 5.04 \\
$\mathrm{NaNO}_{3}$ & 295.1 & 28.8 & 4.46 \\
$\mathrm{KNO}_{3}$ & 329.0 & 14.7 & 3.57 \\
$\mathrm{RbNO}_{3}$ & 341.8 & 11.4 & 3.24 \\
$\mathrm{CsNO}_{3}$ & 333.5 & 9.66 & 3.21
\end{tabular}

As appears from the table, electrolytes with larger ions have lower conductance values and larger dissociation constants. The ion diameters are reasonably in accord with atomic dimension. The reversal of size in the case of the alkali metal ions is doubtless due to solvation.

5. Ion-dipole interactions. Before proceeding further, it will be helpful if we examine the conductance curves of an electrolyte as a function of concentration in solvent media over a wide range of the dielectric constant. Conductance curves are shown in Fig. 6 for solutions of tetraisoamylammonium nitrate in mixtures of dioxane and water whose dialectric constants vary from 2.2 (dioxane) to 78 (water). Logarithms of concentration and of equivalent conductance are plotted in order that all values may be represented in a single figure. The concentration varies from approximately $0.1 \mathrm{~N}$ to $1 \times 10^{-5} \mathrm{~N}$, and the conductance from 100 for water to $3 \times 10^{-5}$ for dioxane. The first two curves $(D=78$ and 37$)$ are normal, approximating the Debye-Hückel-Onsager equation. The third curve $(D=12)$ shows a large conductance decrease; here ion association is beginning to occur. With the fourth curve $(D=9)$, a minimum appears in the curve at about $0.03 \mathrm{~N}$. As the dielectric constant diminishes, the conductance values fall and the minimum shifts to lower concentrations, reaching $2.5 \times 10^{-5}$ in pure dioxane. For dilute solutions, in mixtures having values of $D$ less than 6 , the curves approach limiting tangents with a common slope of $-1 / 2$. This corresponds to a mass action equilibrium between free and associated ions of a 
largely associated electrolyte ( $\gamma$ small). The complexity of the lowermost curves at concentrations above that of the minimum point are of much interest. I shall refer to them again.

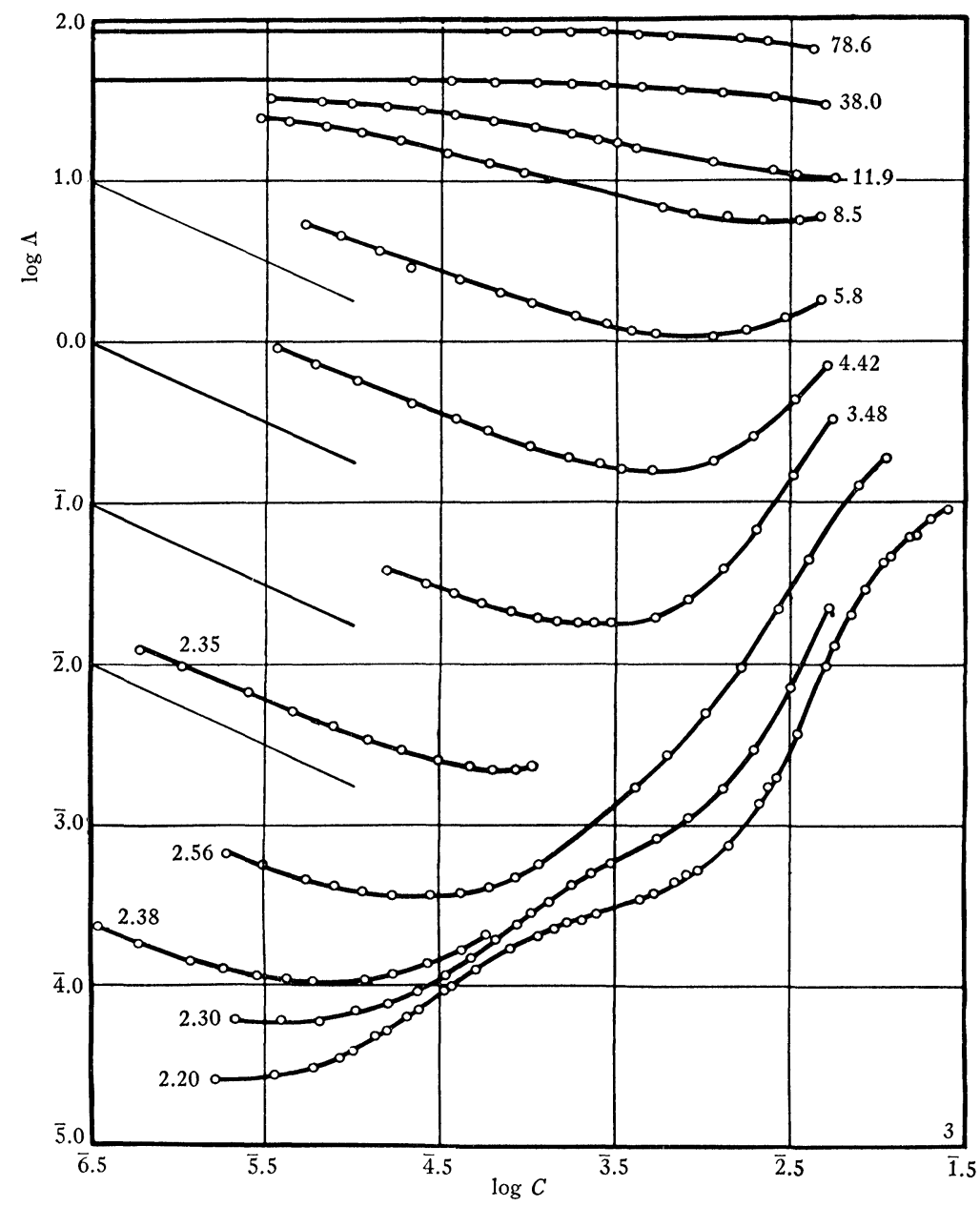

FIG. 6

Plot of $\log \Lambda$ as function of $\log C$ for solutions of tetraisoamylammonium nitrate in water-dioxane mixtures of varying dielectric constants.

The minima in the conductance curves are due to interaction between the simple ions and the associated ions or ion pairs. Thus, if we have an electrolyte $A B$, dissociating into the ions $A^{+}$and $B^{-}$, the ions $A^{+}$and $B^{-}$will interact with the ion pairs $A B$. An equilibrium 
exists between them according to the equations $A B+A^{+}=A B A^{+}$ and $A B+B^{-}=B A B^{-}$. This interaction has been treated by Dr. Fuoss in much the same manner as that between ions and ions. It leads to the following simple equation for the conductance as a function of concentration:

$$
\Lambda C^{1 / 2} g(C)=\Lambda_{0} K^{1 / 2}+\frac{\lambda_{0} K^{1 / 2}}{k}\left(1-\frac{\Lambda}{\Lambda_{0}}\right) C .
$$

Here $g(C)$ is a correction factor taking into account the activity and the mobility terms of the ions; $\lambda_{0}$ is the limiting conductance of the triple ions $A B A^{+}$and $B A B^{-}$, assumed to be equal; and $k$ is the dissociation constant of the triple ion equilibria. Since $\Lambda / \Lambda_{0}$ is small in comparison with unity, a plot of the $\Lambda C^{1 / 2} g(C)$ against $C$ should yield a linear relation. A plot of this kind is shown in Fig. 7 for solutions of tetrabutylammonium picrate in anisole. As may be seen from the

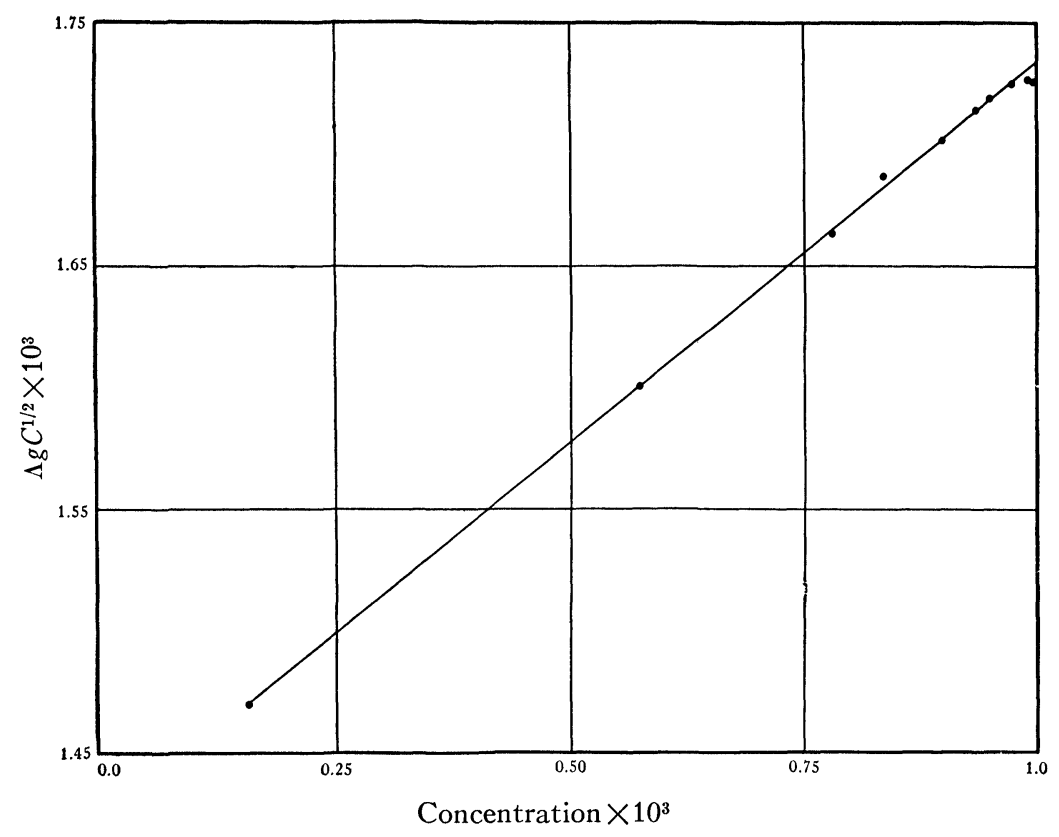

FIG. 7

Plot of conductance function for tetrabutylammonium picrate in anisole showing effect of triple-ion equilibrium.

figure, the experimental points lie upon the required straight line well within the limit of experimental error. At higher concentrations, 
slightly above the concentration at the minimum point, deviations become appreciable and increase with increasing concentration.

6. The effect of potential. An ion moving under the action of a constant applied field is retarded owing to the asymmetry of its ion atmosphere. On the one hand, as we have seen, a potential develops opposing the potential of the field, and, on the other hand, the electrophoretic effect of the ion atmosphere tends to move the solvent in a direction opposite to that of the motion of the ion. Since the time required to build up the ion atmosphere is finite, the condition arises, when the applied field becomes sufficiently strong, that the ion moves away from its atmosphere more rapidly than it (the ion atmosphere) can be built up. In the limiting case, the ion moves quite free of an ion atmosphere and it is in the same condition as it would be if the solution were infinitely dilute. Since the relaxa ion time of the ion atmosphere varies inversely as the concentration, this field effect is greater (for a given potential) the lower the concentration of the solution. This effect was discovered by Wien and has been studied extensively by him and his collaborators; the results are in accord with theory.

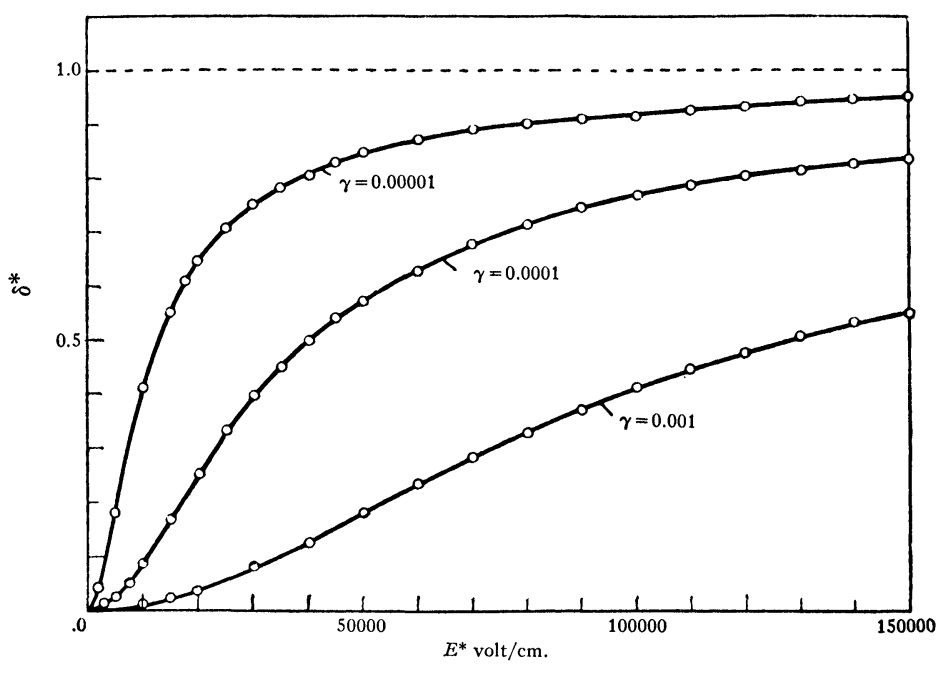

$\gamma=$ Concentration in moles per liter.

FIG. 8

Illustrating the Wien potential effect for solutions of strong electrolytes in water.

The effect of potential on the conductance of aqueous solutions of strong electrolytes is illustrated in Fig. 8, where applied potentials are plotted as abscissas against the increase of conductance due to the 
field divided by the maximum increase possible due to an infinite field. Since the relaxation time is an inverse function of concentration, the conductance change on applying the field reaches its limiting value much more rapidly at low concentrations than at high concentrations.

In the case of weak electrolytes, another effect (due to the field) appears. Owing to the field which tends to separate the oppositely charged ions in their ion pairs, the dissociation of the electrolyte is increased as the field increases. This effect has also been studied by Wien, and the theory has been developed by Onsager.

In Fig. 9 are shown curves for the percentage conductance change of several weak acids in aqueous solution. The experimental points are indicated and the straight lines, as drawn, represent the course of the curves required by theory.

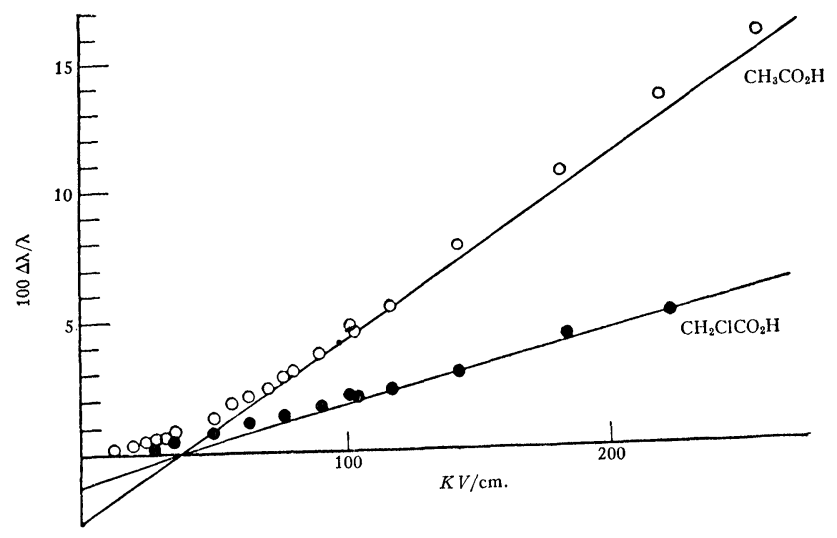

FIG. 9

Illustrating the Wien potential effect for solutions of weak electrolytes in water.

7. Frequency effect. The effect of potential on conductance led Debye and Falkenhagen to suspect an effect due to the frequency of the applied field. They developed a theory which was later confirmed experimentally. The effect is due to the fact that a finite time is required for the asymmetry of the ion atmosphere around a moving ion to disappear when the field is removed. With a slowly alternating field the effect is inappreciable, but when the period of the field approaches the relaxation time of the ion atmosphere, the asymmetry effect of the ion atmosphere on conductance diminishes and disappears at sufficiently high frequencies because the displacement of the central ion in its atmosphere becomes inappreciable in the period of 
an oscillation. The electrophoretic effect due to the ion atmosphere itself remains.

This conductance change over that of the conductance in a stationary field decreases with decreasing concentration. It likewise decreases with decreasing number of charges on the central ion. If the conductance of an electrolyte is measured at a given frequency comparable with the relaxation time of the ions at intermediate concentrations, then, at very low concentrations where the radius of the ion atmosphere is very large, the frequency effect will be zero, since the ions will already have a conductance equal to that of their limiting value for an infinitely dilute solution. With increasing concentration, the conductance change due to frequency increases, passes through a maximum, and thereafter decreases. The reason for the decrease is that the frequency of the applied field is now below that required in order to prevent the building up of the asymmetry of the ion atmosphere.

In Fig. 10 are shown curves for the percentage change in the conductance of magnesium sulfate and manganese sulfate in aqueous solution for a field frequency corresponding to a wave length of one

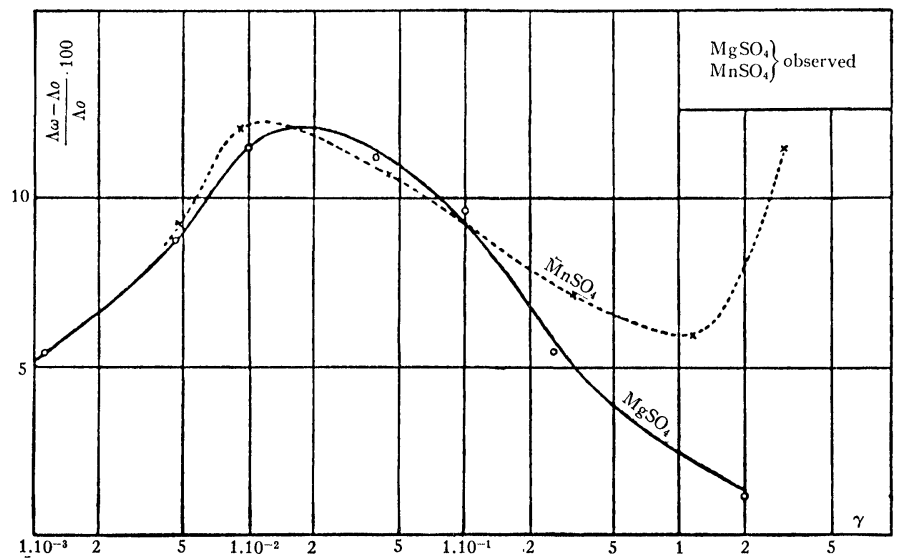

FIG. 10

Illustrating the frequency effect for solutions of salts in water.

meter. For magnesium sulfate, the curve approximates closely to the theoretical value over the entire range of concentration; for manganese sulfate, a large deviation occurs at the higher concentrations. In the case of simpler salts, such as uni-univalent salts, the correspondence between theory and experiment is even better than in that of the two salts above. 
8. Ion dipoles. In measuring the conductance of solutions, we obtain information relating directly to the ions, but our knowledge with respect to the associated ions or ion pairs depends upon inference. It is possible, however, to carry out measurements in which the property measured depends directly upon the ion pairs and not upon the ions.

The dielectric constant of a vacuum is unity; that of all material media is greater than unity. This is due to the fact that the charges in the atoms of molecules of the medium suffer displacement under the action of an impressed potential. With non-polar molecules, this displacement of charge is extremely small, but if the centers of charge of positive and negative electricity in a molecule are not coincident (polar molecules), then the molecule has an electric moment, and it will tend to rotate under the action of the field so as to align the axis of its electrical dipole with the axis of the field. The presence of such dipole molecules in a solvent medium tends to increase the dielectric constant of the solution over that of the pure medium.

If the charges in the dipole are actually separated, as we should expect them to be in the case of associated ions, or ion dipoles, as we may call them, then we should expect that the effect of these dipoles upon the dielectric constant would be very large. The extent of the displacement of charge is stated in terms of the molecular polarization of the dissolved dipoles, and from the polarization we may compute the electric moment (charge times distance) of the dipoles. If, in the dipole molecules, the charges are actually separated, as they are in the case of ionic dipoles, then by dividing the electric moment by the unit charge on the ions, we obtain therefrom the distance of separation of the charges.

In Fig. 11 are shown the polarization curves of a number of electrolytes as derived from dielectric constant measurements of their solutions in benzene. These polarization curves are in part linear and slightly inclined, and in part they show a much higher degree of complexity. The inclination of the linear curves is probably due to the association of the dipoles to form quadrupoles. The rapid diminution of the polarization in the case of other electrolytes is doubtless due to a high degree of association of the dipoles to more complex molecules.

What is striking, however, is the fact that the values of the polarization for electrolytes dissolved in benzene are approximately ten times as great as those of ordinary polar molecules in the same solvent. These high values of the polarization indicate that the charges are separated by considerable distances. Values of the polarization, of 


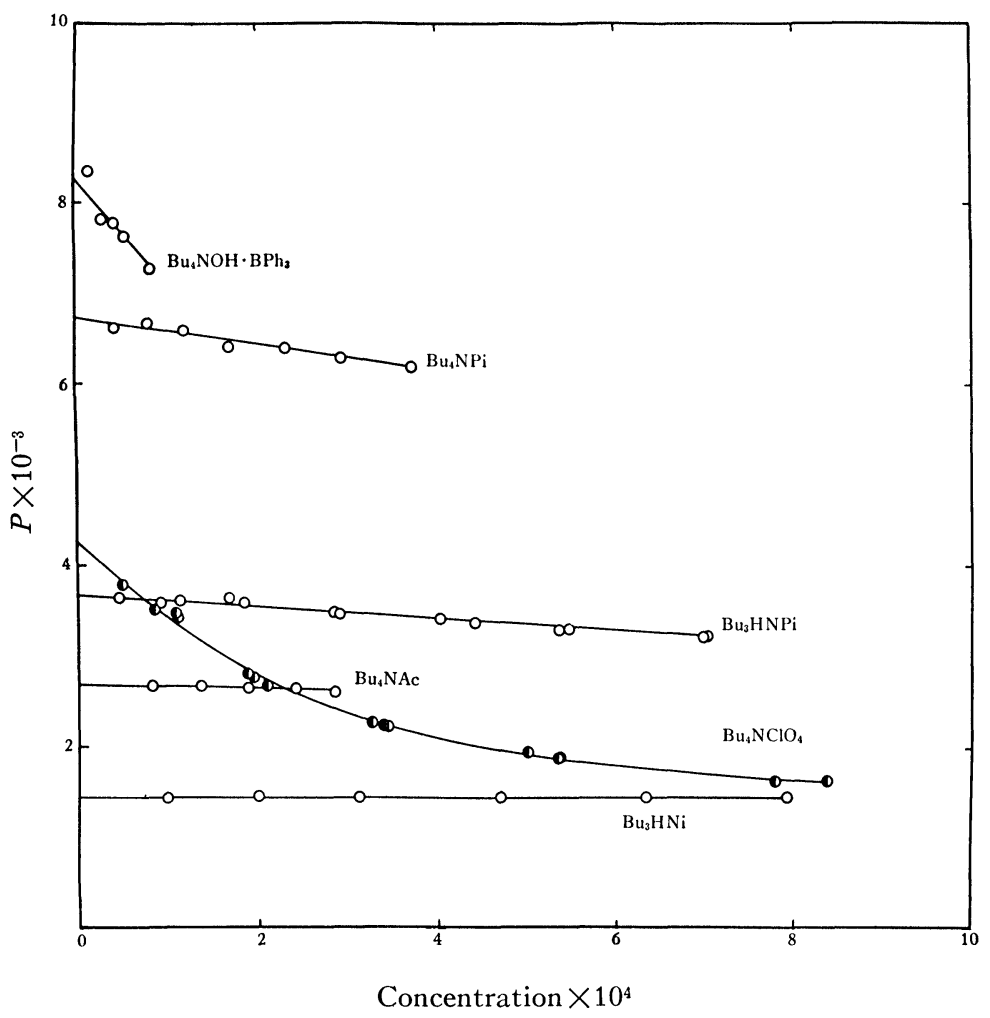

FIG. 11

Polarization curves of electrolytes in benzene solution.

the dipole moments, and of the distance between charges, as computed from the dipole moments, are given in Table 2.

TABLE 2

Electric moments of ion dipoles in benzene

\section{Salt}

$\mathrm{Bu}_{4} \mathrm{~N} \cdot \mathrm{Ac}$

$\mathrm{Bu}_{4} \mathrm{~N} \cdot \mathrm{Br}$

$\mathrm{Bu}_{3} \mathrm{NH} \cdot \mathrm{Pi}$

$\mathrm{Am}_{3} \mathrm{NH} \cdot \mathrm{Pi}$

$\mathrm{Am}_{4} \mathrm{~N} \cdot \mathrm{SCN}$

$\mathrm{Bu}_{4} \mathrm{~N} \cdot \mathrm{Pi}$

$\mathrm{Am}_{4} \mathrm{~N} \cdot \mathrm{Pi}$

$\mathrm{Am}_{4} \mathrm{~N} \cdot \mathrm{HOBPh}_{3}$

$\begin{array}{cc}\text { Polarization } & \text { Moment } \times 10^{18} \\ 2690 & 11.2 \\ 3250 & 12.3 \\ 3770 & 13.2 \\ 3830 & 13.3 \\ 5050 & 15.4 \\ 6740 & 17.8 \\ 7090 & 18.3 \\ 8200 & 19.6\end{array}$

$a \times 10^{8}$

2.34

2.58

2.78

2.80

3.23

3. 74

3.83

4.12

$\mathrm{Bu}=$ butyl, $\mathrm{Am}=$ amyl, $\mathrm{Ph}=$ phenyl, $\mathrm{Ac}=$ acetate, $\mathrm{Pi}=$ picrate . 
On inspection of the table, it will be seen that the distance between charges varies from 2.3 to 4.1 Ångström units $\left(\AA=1 \times 10^{-8} \mathrm{~cm}\right.$.). These values are in reasonably good accord with what we know of molecular and atomic dimensions. It is particularly interesting to note that as the ions become more complex, and therefore larger, the distance between the ions increases.

9. Molecular weights. The decrease in the molecular polarization of electrolytes in benzene with increasing concentration indicates the association of dipoles to quadrupoles and perhaps more complex structures. Dielectric constant measurements, however, can give us no direct evidence as to the existence of these structures. Some further light is thrown on the phenomenon of association by measurements of the molecular weights of electrolytes in benzene. While the force between ions and ions falls off as the inverse square of the distance, and that between ions and dipoles as the inverse cube of the distance, the force between dipoles and dipoles falls off as the inverse fourth power of the distance. We should not, therefore, expect to find a high degree of dipole association in solvents of higher dielectric constant; but in solvents of very low dielectric constant, such as benzene, we should expect association of dipoles to occur. Indeed, we might look for association complexes of higher order, including charged as well as uncharged complexes. If dipole association occurs, measurement of the molecular weight of the dissolved electrolyte should indicate, approximately, its extent. In this connection, it may be noted that the concentration of ions in solutions of electrolytes in benzene is so low that the ion atmosphere effect may be neglected.

In Fig. 12 are shown curves in which are plotted values of the

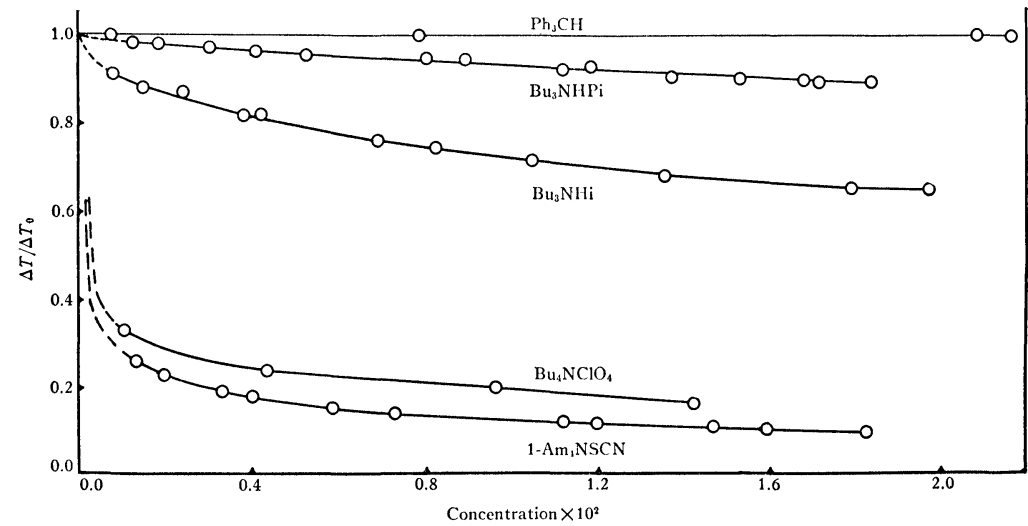

FIG. 12

Freezing point curves of solutions of electrolytes in benzene. 
ratio $\Delta t / \Delta t_{0}$ of the observed freezing point lowering to the lowering of an ideal solute having the same (stoichiometric) concentration.

It will be observed that in the case of all electrolytes the curves lie below that of an ideal solute, indicating a measurable amount of association. An analysis of the curve for tributyl-ammonium picrate (the least associated) indicates that there exists an equilibrium between dipoles and quadrupoles in accordance with the law of mass action. In the case of other electrolytes, association takes place to a larger extent, and the observed values cannot be accounted for on the assumption of a simple equilibrium between dipoles and quadrupoles. Evidently, in these cases, higher complexes than quadrupoles are formed. This is particularly true of tetraisoamylammonium thiocyanate, where the mean molecular weight of the solute at a concentration of $0.017 N$ is eleven times its formula weight. There can be no doubt but that very complex aggregates are formed in solutions of this electrolyte.

The results of these molecular weight determinations are in accord with those of dielectric constant measurements. It will be recalled that the molecular polarization of tetraisoamylammonium thiocyanate falls off very sharply with increasing concentration. The results are also in accord with the results of conductance measurements. In Fig. 6, for example, for tetraisoamylammonium nitrate, the conductance curve for low values of $D$ shows several inflection points at concentrations above the minimum; there is a certain region where the conductance increases very slowly with concentration. A similar curve in the case of tetraisoamylammonium thiocyanate shows an even more marked effect; there is a considerable region where there is scarcely any increase in conductance as the concentration is increased. Presumably, what is happening here is the formation of a large proportion of highly complex neutral structures which, while taking no part in conductance, tend to reduce the normal increase of ions with increasing concentration.

Since in all cases the conductance ultimately rises very rapidly (at high concentrations), it follows that in these regions there must be a rapid increase in the number of ions. Seemingly, there is an equilibrium among a great variety of complex ions and neutral molecules which, with increasing concentration, is shifted in the direction of an increased number of charged structures, that is, structures in which there is a preponderance of either positive or negative ions.

10. Conclusion. The preceding discussion will have shown that the theory based on the assumption of Coulombic interaction between 
ions gives an uncommonly satisfactory account of the properties of electrolytic solutions in the limiting case of dilute solutions. It must not be forgotten, however, that the theory is strictly a limiting theory, and that as yet we have no adequate theory of more concentrated solutions. While a large mass of data is available with respect to concentrated solutions, an adequate theory is lacking. That the present theory may be expanded in some simple fashion so as to account for the more concentrated solutions seems doubtful. It appears more probable that an entirely new method of attack will have to be devised.

Actually, only a beginning has been made in the solution of the problem of electrolytes. The nature of the ions themselves remains unknown. We know that ions in water are hydrated, but the extent of the hydration is not known. There is evidence indicating that ions in media other than water are solvated, but again the extent of solvation remains unknown.

It is known that the conductance of an electrolyte in a given medium is roughly proportional to the fluidity of the medium, but the precise relation between conductance and viscosity remains undetermined. So also the relation between the conductance phenomenon and temperature remains very obscure. Indeed, with the exception of the measurements carried out by A. A. Noyes and his co-workers thirty years ago, we have practically no data with respect to aqueous solutions at temperatures above $100^{\circ}$, and even at that temperature the data are meagre and uncertain. In solvents other than water, there is almost a complete lack of data over any considerable temperature range.

The generality of electrolytic phenomena is still not recognized by many investigators. Practically all liquid media are capable of dissolving suitable electrolytes and yielding solutions that conduct the electric current. Much work remains to be done with solvents of very low dielectric constant. Data with respect to solutions in solvents of dielectric constant higher than that of water are also lacking.

Many substances which are not themselves electrolytes in the pure state yield electrolytic solutions when dissolved in suitable solvents. Such is the case with the acids and many bases in water. Even today we have no sound theory to account for the influence of constitution upon the strength of acids and bases. The properties of acids and bases in solvents other than water remain uninvestigated. So also the properties of the weaker salts in solvents other than water remain unknown. The investigation of the remarkable solutions of the alkali metals in liquid ammonia has been carried only far enough to show 
that these solutions form a link between metallic and electrolytic conductors. These solutions offer a tempting field for further research. Electrolytic solutions find applications in many diverse fields of science, particularly in the biological sciences. Biological systems, in general, are electrolytic systems and many biological phenomena directly involve electrolytes. It does not seem probable that biological processes may be accounted for merely on the basis of what is known of aqueous solutions. Cell structures consist of anything but water and yet these cell structures are of primary importance in connection with many electrolytic phenomena that accompany biological change. The transmission of nerve impulses is recognized to be an electrical phenomenon. Clearly, the electric impulse in this case is not a metallic one; the process must, necessarily, be an electrolytic one. An understanding of the mechanism of such phenomena will be had only when the nature of electrolytic processes in cells is more thoroughly understood.

Brown UNIVERSITY 